\title{
The potential pathogenicity of chlorhexidine- sensitive Acanthamoeba strains isolated from contact lens cases from asymptomatic individuals in Tenerife, Canary Islands, Spain
}

Correspondence Basilio Valladares bvallada@ull.es

Received 13 May 2008 Accepted 15 July 2008

\author{
Carmen M. Martín-Navarro, Jacob Lorenzo-Morales, \\ M. Gabriela Cabrera-Serra, Fernando Rancel, Nieves M. Coronado-Álvarez, \\ José E. Piñero and Basilio Valladares
}

University Institute of Tropical Diseases and Public Health of the Canary Islands, University of La Laguna, Avda. Astrofísico Fco. Sánchez, S/N, 38203 La Laguna, Tenerife, Canary Islands, Spain

\begin{abstract}
Pathogenic strains of the genus Acanthamoeba are causative agents of a serious sightthreatening infection of the eye known as Acanthamoeba keratitis. The prevalence of this infection has risen in the past 20 years, mainly due to the increase in number of contact lens wearers. In this study, the prevalence of Acanthamoeba in a risk group constituted by asymptomatic contact lens wearers from Tenerife, Canary Islands, Spain, was evaluated. Contact lenses and contact lens cases were analysed for the presence of Acanthamoeba isolates. The isolates' genotypes were also determined after rDNA sequencing. The pathogenic potential of the isolated strains was subsequently established using previously described molecular and biochemical assays, which allowed the selection of three strains with high pathogenic potential. Furthermore, the sensitivity of these isolates against two standard drugs, ciprofloxacin and chlorhexidine, was analysed. As the three selected strains were sensitive to chlorhexidine, its activity and $\mathrm{IC}_{50}$ were evaluated.

Chlorhexidine was found to be active against these strains and the obtained $\mathrm{IC}_{50}$ values were compared to the concentrations of this drug present in contact lens maintenance solutions. It was observed that the measured $\mathrm{IC}_{50}$ was higher than the concentration found in these maintenance solutions. Therefore, the ineffectiveness of chlorhexidine-containing contact lens maintenance solutions against potentially pathogenic strains of Acanthamoeba is demonstrated in this study.
\end{abstract}

\section{INTRODUCTION}

Free-living amoebae of the genus Acanthamoeba are ubiquitous protozoans that pervade the entire environment and include amphizoic strains that are pathogenic to humans and animals (Schuster \& Visvesvara, 2004a). These protozoans are opportunistic causal agents of a sightthreatening ulceration of the cornea called Acanthamoeba keratitis (AK), disseminated infections (mostly cutaneous and nasopharyngeal) and a usually fatal granulomatous amoebic encephalitis (Khan, 2003, 2006; Marciano-Cabral \& Cabral, 2003; Schuster \& Visvesvara, 2004a).

$\mathrm{AK}$ in developed countries is often associated with inadequate care of the contact lenses and also as a consequence of corneal trauma whereas in developing

Abbreviations: AK, Acanthamoeba keratitis; PHMB, polyhexamethylene biguanide.

The GenBank/EMBL/DDBJ accession numbers for the diagnostic fragment 3 sequence of the 28 new Acanthamoeba isolates are EU686695-EU686722. nations most cases occur as a result of ocular trauma (Seal et al., 1999; Ibrahim et al., 2007; Ozkoc et al., 2008). AK symptoms are nonspecific and can be misdiagnosed as a viral, bacterial or fungal keratitis. Thus an early diagnosis is required to achieve a successful therapeutic outcome (Martínez \& Visvesvara, 1991; Lorenzo-Morales et al., 2007). Currently, the recommended treatment regimen includes a biguanide $[0.02 \%$ polyhexamethylene biguanide (PHMB) or $0.02 \%$ chlorhexidine digluconate] together with a diamidine $(0.1 \%$ propamidine isethionate, also known as Brolene, or $0.1 \%$ hexamidine, also known as Desomedine) (Khan, 2006). Biguanides are most often used due to their excellence in the treatment of AK and are frequently combined with a diamidine due to their presumed additive anti-amoebic effect (Hay et al., 1994; Schuster \& Visvesvara, 2004b; Lim et al., 2008). Moreover, chlorhexidine and PHMB as monotherapy agents at normal concentration have been proven not to be sufficient against clinical or environmental strains of acanthamoebae and this has highlighted the importance of multiple-strain testing of drugs against acanthamoebae as their effective- 
ness might depend on the acanthamoeba isolate (Lim et al., 2008; Gooi et al., 2008; Shoff et al., 2008).

It is also important to mention that drugs to be used for the treatment of AK should be both amoebicidal and cysticidal as there is a risk of relapse due to the re-emergence of amoebae from the more resistant cyst (Schuster \& Visvesvara, 2004b). Chlorhexidine has been shown to be active against both Acanthamoeba trophozoites and cysts, as it interferes with the mucopolysaccharides of the cyst at the ostiole level and also in the lipid bilayer of the cellular membrane, causing cellular lysis (Lim et al., 2008).

The taxonomy and classification of these protozoans are continually under revision, following the successful application of molecular techniques (Stothard et al., 1998; Booton et al., 2002, 2005). Evolutionary studies have led to the identification of 15 different genotypes (T1-T15) based on rRNA gene sequencing (Stothard et al., 1998; Horn et al., 1999; Gast, 2001; Hewett et al., 2003). The correlation between pathogenicity and certain genotypes continues to be investigated (Stothard et al., 1998; Gast, 2001; Booton et al., 2005; Maghsood et al., 2005). To date, studies have shown that $90 \%$ of Acanthamoeba isolates that produce infections belong to the T4 genotype, suggesting that the abundance of T4 isolates in human infections is most likely due to their greater virulence and/or properties that enhance their transmissibility as well as their decreased susceptibility to chemotherapeutic agents (Maghsood et al., 2005; Khan, 2006).

A role for extracellular proteases, mainly serine, metalloand cysteine proteases, in the pathogenicity of Acanthamoeba has been suggested previously (Khan, 2003), and higher quantities of extracellular proteases from pathogenic compared to non-pathogenic strains seem to be responsible for epithelial-cell destruction. Serine protease activity is likely to be a major factor in the pathogenesis of amoebic keratitis or granulomatous amoebic encephalitis consequential to Acanthamoeba infection (Lorenzo-Morales et al., 2005a, c; Khan, 2006) as these proteases have shown an enhanced capacity to degrade proteins such as collagen, fibronectin, laminin, sIgA, IgG, plasminogen, fibrinogen and haemoglobin $(\mathrm{Na}$ et al., 2001). It is important, therefore, to study the secretion of proteases as this allows the differentiation between pathogenic and non-pathogenic Acanthamoeba strains depending on the type, number and the degree of secretion (Khan, 2003; Lorenzo-Morales et al., 2005a, c).

In this work, a number of different Acanthamoeba strains were isolated from contact lens cases from asymptomatic individuals in Tenerife, Canary Islands, Spain. Certain strains which were indicated to be particularly pathogenic by a number of criteria were further tested with respect to their sensitivity to chlorhexidine and ciprofloxacin. Chlorhexidine was demonstrated to be active against these strains, but the concentration of chlorhexidine required to kill these strains was higher than those found in commercial contact lens maintenance solutions.

\section{METHODS}

Isolation and cultivation. One hundred and fifty-three contact lens cases (90 of them included their contact lenses) from asymptomatic immunocompetent individuals from Tenerife, Canary Islands, Spain, were included in this study. Data regarding the type of contact lens, disinfectant systems, length of wear and contact lens hygiene were collected together with the contact lens cases and contact lenses.

Contact lens cases were washed using sterile saline solution. The obtained washings were filtered through a $0.45 \mu \mathrm{m}$ pore diameter cellulose nitrate filter (Millipore) with a weak vacuum (flow rate $1.3 \mathrm{ml} \mathrm{min}{ }^{-1}$ ). These membranes were then cultured in $2 \%$ nonnutrient agar (NNA) covered with heat-killed Escherichia coli and incubated at $28{ }^{\circ} \mathrm{C}$ to allow growth of non-pathogenic and pathogenic amoebal strains. After 3-4 days, the membranes were removed and the plates were incubated at $28{ }^{\circ} \mathrm{C}$ for a further $1-2$ weeks. Swabs were then stroked on NNA plates within an area 40 by $40 \mathrm{~mm}$ in size and incubated at $28{ }^{\circ} \mathrm{C}$ for up to 2 weeks. These plates were monitored for out-growth of Acanthamoeba microscopically and blocks containing Acanthamoeba were removed and the amoebae were cloned by dilution. Acanthamoeba isolated in this way was then transferred into axenic cultures by placing the amoebae into PYG medium $[0.75 \%(\mathrm{w} / \mathrm{v})$ proteose peptone, $0.75 \%(\mathrm{w} / \mathrm{v})$ yeast extract and $1.5 \%(\mathrm{w} / \mathrm{v})$ glucose] containing $40 \mu \mathrm{g}$ gentamicin $\mathrm{ml}^{-1}$ (Biochrom). Genus-specific PCR was carried out in order to confirm the positive cultures as previously described (Vodkin et al., 1992).

Characterization of the pathogenic potential. The pathogenic potential of the isolated amoebae was evaluated using extracellular protease zymograms and assays to determine thermotolerance and osmotolerance, as previously described (Khan et al., 2000; LorenzoMorales et al., 2005c). Briefly, SDS-PAGE gels containing gelatin $\left(2 \mathrm{mg} \mathrm{ml}^{-1}\right)$ were used for the analysis of extracellular proteases and the type of secreted protease was examined by using protease inhibitors such as PMSF (serine protease inhibitor, $2 \mathrm{mM}$ final concentration), E-64 (cysteine protease inhibitor, $5 \mu \mathrm{m}$ final concentration) or EDTA (metalloprotease inhibitor, $10 \mathrm{mM}$ final concentration) for $30 \mathrm{~min}$ prior to electrophoresis.

For temperature tolerance assays, NNA plates seeded with E. coli were inoculated with approximately 1000 cells, and the plates were incubated at $28{ }^{\circ} \mathrm{C}, 37{ }^{\circ} \mathrm{C}$ and $44{ }^{\circ} \mathrm{C}$ for up to $72 \mathrm{~h}$. To examine the effect of osmolarity on the growth of Acanthamoeba trophozoites, NNA plates containing $0.1 \mathrm{M}, 1.0 \mathrm{M}$ and $1.5 \mathrm{M}$ mannitol were seeded with E. coli as previously described (Khan et al., 2001). Approximately 1000 trophozoites were inoculated onto these plates and incubated at $28{ }^{\circ} \mathrm{C}$ for up to $72 \mathrm{~h}$. Growth and the survival rate of amoebae were checked during this period and resistance to all assayed conditions classified the amoebae as thermo- and osmotolerant as described previously (Khan et al., 2001).

Moreover, PCR of the serine protease catalytic domain (Hong et al., 2000) was also performed as previously described as this assay together with the zymograms and thermo- and osmotolerance assays could determine the pathogenic potential of these amoebae (LorenzoMorales et al., 2005b, c).

Activity assays. The activity of two drugs was tested on selected strains of Acanthamoeba from this study: chlorhexidine (chlorhexidine digluconate; Alfa Aesar), a standard antiseptic belonging to the biguanide family of compounds which are commonly used in contact lens maintenance solutions; and ciprofloxacin (ciprofloxacin lactate; Dr. Ehrenstorfer), an antibiotic that has been previously used for the treatment of AK (Colin \& Hasle, 1993; Wynter-Allison et al., 2005). For the sensitivity and activity assays, a type strain from the American Type Culture Collection, Acanthamoeba castellanii (Neff strain) ATCC 30010, genotype T4, was used as a control. 
For the activity assays, a previously developed colorimetric 96-well microtitre plate assay, based on the oxidoreduction of alamarBlue, was used for the determination of drug efficacy against the trophozoites of the selected Acanthamoeba strains as previously described by McBride et al. (2005). Subsequently the plates were analysed, over a period of $72-120 \mathrm{~h}$, on a Microplate Reader model 680 (Bio-Rad) using a test wavelength of $570 \mathrm{~nm}$ and a reference wavelength of $630 \mathrm{~nm}$. For those strains that were sensitive to the assayed drugs, the percentage of inhibition and $50 \%$ inhibitory concentrations $\left(\mathrm{IC}_{50}\right)$ were calculated by linear regression analysis using a $95 \%$ confidence limit. All experiments were performed three times each in duplicate and the mean values were also calculated. A paired two-tailed $t$-test was used for analysis of the data. Values of $P$ $<0.05$ were considered significant. The inhibition curves of the statistical analysis were developed using the Sigma Plot 9.0 software program (Systat Software).

Genotyping of Acanthamoeba isolates. Acanthamoeba spp. genotype determination was based on sequence analysis of diagnostic fragment 3 (DF3), a subset of the nuclear small subunit rRNA gene as previously described. Obtained sequences were compared to sequences available in GenBank and genotypes of the new isolates were calculated by $<5 \%$ dissimilarity as described previously (Stothard et al., 1998; Booton et al., 2002, 2005). Obtained sequences were also compared with the Acanthamoeba DNA sequence database (Department of Molecular Genetics, The Ohio State University, OH, USA). Sequences for the 28 new isolates are deposited in GenBank under the accession numbers EU686695-EU686722.

\section{RESULTS}

We found that $65.9 \%$ of the analysed contact lenses and contact lens cases were positive for Acanthamoeba. Moreover, $30 \%$ of the isolated amoebae were found to be potentially pathogenic and secreted extracellular serine proteases.

When the type of contact lens was evaluated, $81 \%$ of the individuals in this study were monthly soft contact lens wearers. Furthermore, $52 \%$ of the contact lenses were positive for Acanthamoeba, and $30 \%$ of these amoebae were potentially pathogenic.

Regarding genotyping results, it was revealed that the isolated Acanthamoeba strains belonged to the T1 (3.6\%), T3 (14.3\%), T4 (78.6\%) and T10 (3.6\%) genotypes.

The most common maintenance solution used by $49 \%$ of the study sample was the so-called 'one-step' system. This finding was important, as $52 \%$ of the 'one-step' users were positive for Acanthamoeba, and $28 \%$ of the strains isolated were determined to be potentially pathogenic (Table 1 ). If the antiseptic content of the maintenance solutions is considered (Fig. 1), $65 \%$ of the individuals in this study were using solutions containing PHMB. Of the latter group, $48 \%$ were positive for Acanthamoeba strains, of which $26 \%$ were potentially pathogenic strains.

Zymograms of extracellular proteases showed that all the isolated amoebae were protease secretors. Additionally, $30 \%$ of the isolates secreted serine proteases, as determined by zymography and PCR amplification of the serine protease catalytic domain, and therefore were considered
Table 1. Percentages of use, lenses positive for Acanthamoeba in each group and percentage of potentially pathogenic strains related to the number of positives found in this work

Data are shown for each type of contact lens included in this study.

\begin{tabular}{|lccc|}
\hline Type of lens & $\begin{array}{c}\text { Use } \\
(\%)\end{array}$ & $\begin{array}{c}\text { Positives } \\
(\%)\end{array}$ & $\begin{array}{c}\text { Potentially } \\
\text { pathogenic (\%) }\end{array}$ \\
\hline Hard & 0 & 0 & 0 \\
Daily soft & 7 & 37 & 0 \\
Monthly soft & 81 & 52 & 30 \\
Bi-monthly soft & 3 & 67 & 50 \\
One-year soft & 4 & 50 & 0 \\
Two-year soft & 5 & 50 & 33 \\
& & & \\
\hline
\end{tabular}

potentially pathogenic. Based on the zymograms and PCR of the serine protease catalytic domain as well as genotyping results, three strains were selected for the sensitivity and activity assays mentioned previously: CLC16 (T3 genotype), CLC-41.r (T4 genotype) and CLC-51.1 (T1 genotype). Moreover, most of the proteases that were secreted by these strains were serine proteases. In the case of CLC-51.1, metalloproteases were also observed (Fig. 2).

All tested strains, CLC-16, CLC-41.r, CLC-51.1 and A. castellanii Neff, were sensitive to ciprofloxacin and chlorhexidine assayed microscopically and colorimetrically. We chose to study the effect of chlorhexidine as it belongs to a family of molecules that are common components of contact lens maintenance solutions. Activity assays with

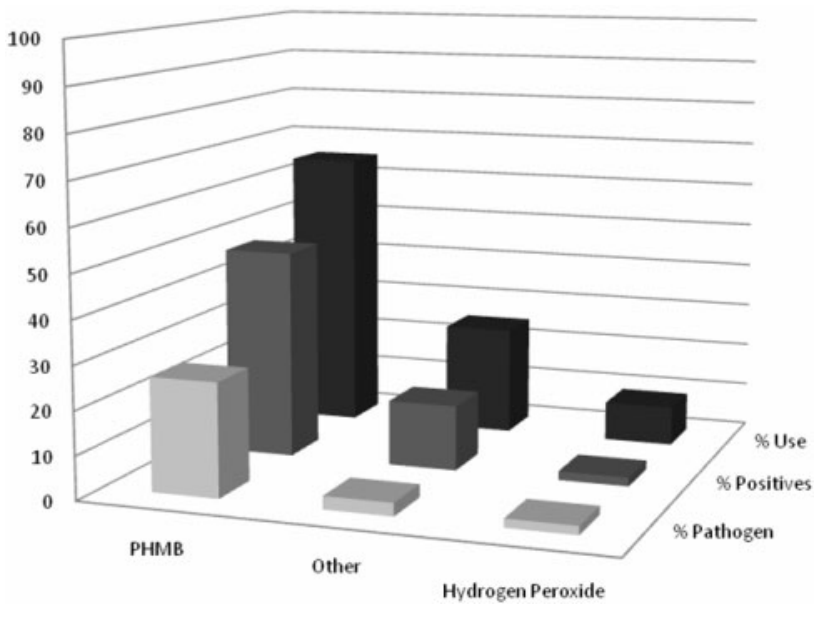

Fig. 1. Percentages of use for different antiseptics in the maintenance solutions of the contact lens wearers included in this study. The most common antiseptic was PHMB. \% Use $=\%$ of contact lens wearers using each type of contact lens; \% positives $=\%$ of these cases where Acanthamoeba was found; $\%$ pathogens $=\%$ of these Acanthamoeba strains that were determined to be likely pathogens through protease and osmotolerance assays. 


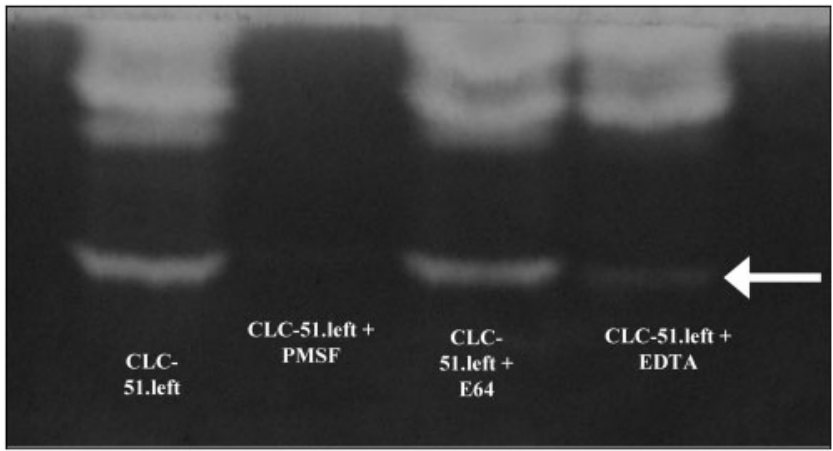

Fig. 2. Protease zymograms of CLC-51.I incubated with different protease inhibitors (PMSF for serine proteases, E-64 for cysteine proteases and EDTA for metalloproteases). Most of the secreted proteases were serine proteases, although evidence of a metalloprotease was also observed, EDTA lane (arrow).

chlorhexidine and the $\mathrm{IC}_{50}$ data for each strain indicated that this compound was able to inhibit the growth of these amoebae even at low concentrations. The $\mathrm{IC}_{50}$ values at $96 \mathrm{~h}$ were $11.89 \pm 3.80, \quad 2.45 \pm 0.20, \quad 10.47 \pm 1.48$ and $5.23 \pm 0.55 \mu \mathrm{M}$ for CLC-16, CLC-41.r, CLC-51.1 and the type strain $A$. castellanii Neff, respectively.

\section{DISCUSSION}

Over the last few decades, AK has become increasingly recognized as important in human health. With an increasing number of people wearing contact lenses, it is important to assess any associated risks, and to make both existing and new users aware of these risks (Khan, 2006). In this study, the number of contact lens wearers that were positive for Acanthamoeba was much higher than in other studies. In a smaller study based in the south-west of Scotland, UK, Acanthamoeba were isolated from 46 (2.2\%) contact lens storage cases tested (Seal et al., 1999). A previous larger study found Acanthamoeba in 4 out of 178 $(2.2 \%)$ lens cases from asymptomatic users in the same region (Devonshire et al., 1993). The reason for the large difference in number is presently unknown but may be connected to the warm climate and dust encountered in Tenerife.

The AK incidence rate also varies between different geographical locations, and these variations are most likely related to extended wear of soft contact lenses, lack of awareness of the potential risks associated with wearing contact lenses, enhanced detection and/or local conditions that promote growth of pathogenic amoebae such as water hardness or salinity (Seal et al., 1999; Radford et al., 2002; Khan, 2006). The latter is highly important in relation to this study as potentially pathogenic strains of Acanthamoeba, including strains belonging to T4 genotype, were previously isolated from soil and water sources on the island of Tenerife (Lorenzo-Morales et al., 2005b). We found that $78.6 \%$ of the strains isolated in this study belonged to genotype T4, confirming previous reports that $\mathrm{T} 4$ is the most common genotype worldwide (Khan, 2006).

The most commonly used antiseptics in contact lens maintenance solutions are PHMB (at concentrations of between $0.0001 \%$ and $0.002 \%$ ) and hydrogen peroxide (maximum $3 \%$ ). However, most solutions also contain hydrant components such as sodium hyaluronate and antifungal agents.

It is also important to mention that PHMB and chlorhexidine (both biguanides) have shown amoebicidal and cysticidal properties against $\mathrm{AK}$ isolates and various type strains of Acanthamoeba (Larkin et al., 1992; Chomicz et al., 2005), and that the clinical outcome is similar for both (Lim et al., 2008). In this study, a high number of users of biguanide-containing (mostly PHMB) maintenance solutions were positive for Acanthamoeba. This could be explained if the obtained $\mathrm{IC}_{50}$ values of chlorhexidine with the strains tested in this study are compared to the concentrations of biguanides (mostly PHMB) present in most maintenance solutions, these being much lower than the concentrations used in this study. The concentrations of biguanides (mostly PHMB) in maintenance solutions are not sufficiently high enough to destroy all amoebic contaminants. Previous studies have demonstrated that disinfectant systems are only effective if recommended hygiene regimes are strictly followed (Borazjani \& Kilvington, 2005). It is also important to mention recent reports regarding the existence of different $\mathrm{T} 4$ strains that have shown diverse resistance levels against anti-amoebic drugs (Shoff et al., 2007). Therefore, multiple-strain testing of drugs against acanthamoebae is recommended as their effectiveness might depend on the isolate (Shoff et al., 2007; Lim et al., 2008; Gooi et al., 2008).

Another important observation in this study was the existence of variations in the incidence of potentially pathogenic Acanthamoeba strains depending on the type of contact lenses and length of use. As suggested above, the major risk factor for AK is poor lens hygiene (Schuster \& Visvesvara, 2004a; Khan, 2006). In support of this statement, more than $85 \%$ of AK cases occur in wearers of contact lenses and this fact is associated with individual behaviour regarding poor personal hygiene, poor handling and care of their lenses or lens storage cases, and noncompliance with disinfection procedures such as using home-made saline or contact with contaminated water sources (Niederkorn et al., 1999; Brennan, 2002; Khan, 2006). These observations were corroborated by the results obtained in this study, as no potentially pathogenic amoebae were isolated from any daily disposable soft contact lens. In contrast, a high number of potentially pathogenic strains were isolated from soft contact lenses worn monthly or bi-monthly. With regards to the monthly use of soft contact lenses, the increased presence of amoebae may be explained by the overuse of the 'onestep' system and the general misuse and the poor hygiene 
adopted for these types of lenses and their cases. It is also important to mention previous observations regarding the lack of efficacy against acanthamoebae of the majority of these one-step solutions (Hiti et al., 2002, 2005; MowreyMcKee \& George, 2007). This risk of poor hygiene and care is obviously increased in the case of contact lenses worn bimonthly as confirmed by the results in this study (Table 1). Finally, the 1-year- or 2-year-use contact lenses showed similar percentages for the presence of acanthamoebae; however, potentially pathogenic Acanthamoeba strains were only isolated from the 2-year-use contact lenses. In the case of 1-year-use lenses, this could be due to the recommendations of complementary care such as 'enzymatic' treatments that commonly contained hydrogen peroxide. Two-year-use contact lenses presented a high percentage of potentially pathogenic amoebae mostly due to a lack of hygiene and poor care of the lenses and their cases (Table 1).

The strains selected for the sensitivity and activity assays were more sensitive to chlorhexidine than to ciprofloxacin. Even so, ciprofloxacin should be considered for a combined therapy as antibiotics can reduce bacterial infection and also eliminate a source of nutrients for acanthamoebae (Khan, 2006). Furthermore, chlorhexidine would be a good candidate for inclusion in contact lens maintenance solutions as it is an inexpensive antiseptic that has been classified as an essential drug by the World Health Organization. Moreover, as previously mentioned, chlorhexidine is active against both trophozoites and cysts of Acanthamoeba, even at minimal concentrations that are not toxic to corneal epithelium cells (Green et al., 1980; Lee et al., 2007).

In conclusion, chlorhexidine, rather than $\mathrm{PHMB}$, or other biguanides should be considered for inclusion in contact lens maintenance solutions, especially in light of the fact that PHMB has been reported to be cytotoxic to human corneal cells (Lee et al., 2007). Additionally, the concentration of chlorhexidine or other biguanides should be reevaluated as the maintenance solutions were shown to be ineffective against the potentially pathogenic strains isolated during this study.

\section{ACKNOWLEDGEMENTS}

C. M. Martin-Navarro was funded by a student research grant from Ministerio de educación y ciencia.

\section{REFERENCES}

Booton, G. C., Kelly, D. J., Chu, Y. W., Seal, D. V., Houang, E., Lam, D. S., Byers, T. J. \& Fuerst, P. A. (2002). $18 \mathrm{~S}$ ribosomal DNA typing and tracking of Acanthamoeba species isolates from corneal scrape specimens, contact lenses, lens cases, and home water supplies of Acanthamoeba keratitis patients in Hong Kong. J Clin Microbiol 40, 1621-1625.

Booton, G. C., Visvesvara, G. S., Byers, T. J., Kelly, D. J. \& Fuerst, P. A. (2005). Identification and distribution of Acanthamoeba species genotypes associated with nonkeratitis infections. J Clin Microbiol 43, 1689-1693.

Borazjani, R. N. \& Kilvington, S. (2005). Efficacy of multipurpose solutions against Acanthamoeba species. Cont Lens Anterior Eye 28, 169-175.

Brennan, N. A. (2002). Is there a question of safety with continuous wear? Clin Exp Optom 85, 127-140.

Chomicz, L., Zebrowska, J., Piekarczyk, J., Starosciak, B. \& Myjak, P. (2005). In vitro studies on susceptibility of Acanthamoeba castellanii to selected chemical agents. Acta Parasitol 50, 25-31.

Colin, J. \& Hasle, D. (1993). Corneal and conjunctival infections. Curr Opin Ophthalmol 4, 54-57.

Devonshire, P., Munro, F. A., Abernethy, C. \& Clark, B. J. (1993). Microbial contamination of contact lens cases in the west of Scotland. Br J Ophthalmol 77, 41-45.

Gast, R. J. (2001). Development of an Acanthamoeba-specific reverse dot-blot and the discovery of a new ribotype. J Eukaryot Microbiol 48, 609-615.

Gooi, P., Lee-Wing, M. \& Brownstein, S. (2008). Acanthamoeba keratitis - persistent organism without inflammation after 1 year of topical chlorhexidine. Cornea 27, 246-248.

Green, K., Livingston, V., Bowmank, K. \& Hull, D. S. (1980). Chlorhexidine effects on corneal epithelium and endothelium. Arch Ophthalmol 98, 1273-1278.

Hay, J., Kirkness, C. M. \& Seal, D. V. (1994). Drug-resistance and Acanthamoeba keratitis - the quest for alternative antiprotozoal chemotherapy. Eye 8, 555-563.

Hewett, M. K., Robinson, B. S., Monis, P. T. \& Saint, C. P. (2003). Identification of a new Acanthamoeba $18 \mathrm{~S}$ rRNA gene sequence type corresponding to the species Acanthamoeba jacobsi Sawyer, Nerad and Visvesvara, 1992 (Lobosea: Acanthamoebidae). Acta Protozool 42, 325-329.

Hiti, K., Walochnik, J., Haller-Schober, E. M., Faschinger, C. \& Aspock, H. (2002). Viability of Acanthamoeba after exposure to a multipurpose disinfecting contact lens solution and two hydrogen peroxide systems. Br J Ophthalmol 86, 144-146.

Hiti, K., Walochnik, J., Faschinger, C., Haller-Schober, E. M. \& Aspock, H. (2005). One- and two-step hydrogen peroxide contact lens disinfection solutions against Acanthamoeba: how effective are they? Eye 19, 1301-1305.

Hong, Y. C., Kong, H. H. \& Ock, M. S. (2000). Isolation and characterization of a cDNA encoding a subtilisin-like serine proteinase (AhSUB) from Acanthamoeba healyi. Mol Biochem Parasitol 111, 441-446.

Horn, M., Fritsche, T. R., Gautom, R. K. \& Wagner, M. (1999). Novel bacterial endosymbionts of Acanthamoeba spp. related to the Paramecium caudatum symbiont Caedibacter caryphilus. Environ Microbiol 1, 357-367.

Ibrahim, Y. W., Boase, D. L. \& Cree, I. A. (2007). Factors affecting the epidemiology of Acanthamoeba keratitis. Ophthalmic Epidemiol 14, 53-60.

Khan, N. A. (2003). Pathogenesis of Acanthamoeba infections. Microb Pathog 34, 277-285.

Khan, N. A. (2006). Acanthamoeba: biology and increasing importance in human health. FEMS Microbiol Rev 30, 564-595.

Khan, N. A., Jarroll, E. L. \& Panjwani, N. (2000). Proteases as markers for differentiation of pathogenic and nonpathogenic species of Acanthamoeba. J Clin Microbiol 38, 2858-2861.

Khan, N. A., Jarroll, E. L. \& Paget, T. A. (2001). Acanthamoeba can be differentiated by the polymerase chain reaction and simple plating assays. Curr Microbiol 43, 204-208. 
Larkin, D. F. P., Kilvington, S. \& Dart, J. K. G. (1992). Treatment of Acanthamoeba keratitis with polyhexamethylene biguanide. Ophthalmology 99, 185-191.

Lee, J. E., Oum, B. S. \& Choi, H. Y. (2007). Cysticidal effect on Acanthamoeba and toxicity on human keratocytes by polyhexamethylene biguanide and chlorhexidine. Cornea 26, 736-741.

Lim, N., Goh, D. \& Bunce, C. (2008). Comparison of polyhexamethylene biguanide and chlorhexidine as monotherapy agents in the treatment of Acanthamoeba keratitis. Am J Ophthalmol 145, 130-135.

Lorenzo-Morales, J., Monteverde-Miranda, C. A. \& Jiménez, C. (2005a). Evaluation of Acanthamoeba isolates from environmental sources in Tenerife, Canary Islands, Spain. Ann Agric Environ Med 12, 233-236.

Lorenzo-Morales, J., Ortega-Rivas, A. \& Foronda, P. (2005b). Isolation and identification of pathogenic Acanthamoeba strains in Tenerife, Canary Islands, Spain from water sources. Parasitol Res 95, 273-277.

Lorenzo-Morales, J., Ortega-Rivas, A. \& Foronda, P. (2005c). RNA interference (RNAi) for the silencing of extracellular serine proteases genes in Acanthamoeba: molecular analysis and effect on pathogenicity. Mol Biochem Parasitol 144, 10-15.

Lorenzo-Morales, J., Martínez-Carretero, E. \& Batista, N. (2007). Early diagnosis of amoebic keratitis due to a mixed infection with Acanthamoeba and Hartmannella. Parasitol Res 102, 167-169.

Maghsood, A. H., Sissons, J., Rezaian, M., Nolder, D., Warhurst, D. \& Khan, N. A. (2005). Acanthamoeba genotype T4 from the UK and Iran and isolation of the T2 genotype from clinical isolates. $J$ Med Microbiol 54, 755-759.

Marciano-Cabral, F. \& Cabral, G. (2003). Acanthamoeba spp. as agents of disease in humans. Clin Microbiol Rev 16, 273-307.

Martínez, A. J. \& Visvesvara, G. S. (1991). Laboratory diagnosis of pathogenic free-living amoebas - Naegleria, Acanthamoeba, and Leptomyxid. Clin Lab Med 11, 861-872.

McBride, J., Ingram, P. R. \& Henriquez, F. L. (2005). Development of colorimetric microtiter plate assay for assessment of antimicrobials against Acanthamoeba. J Clin Microbiol 43, 629-634.

Mowrey-McKee, M. \& George, M. (2007). Contact lens solution efficacy against Acanthamoeba castellanii. Eye Contact Lens 33, 211-215.
Na, B. K., Kim, J. C. \& Song, C. Y. (2001). Characterization and pathogenetic role of proteinase from Acanthamoeba castellanii. Microb Pathog 30, 39-48.

Niederkorn, J. Y., Alizadeh, H. \& Leher, H. (1999). The pathogenesis of Acanthamoeba keratitis. Microbes Infect 1, 437-443.

Ozkoc, S., Tuncay, S. \& Delibas, S. B. (2008). Identification of Acanthamoeba genotype T4 and Paravahlkampfia sp. from two clinical samples. J Med Microbiol 57, 392-396.

Radford, C. F., Minassian, D. C. \& Dart, J. K. G. (2002). Acanthamoeba keratitis in England and Wales: incidence, outcome, and risk factors. Br J Ophthalmol 86, 536-542.

Schuster, F. L. \& Visvesvara, G. S. (2004a). Free-living amoebae as opportunistic and non-opportunistic pathogens of humans and animals. Int J Parasitol 34, 1001-1027.

Schuster, F. L. \& Visvesvara, G. S. (2004b). Opportunistic amoebae: challenges in prophylaxis and treatment. Drug Resist Updat 7, 41-51.

Seal, D. V., Kirkness, C. M. \& Bennett, H. G. (1999). Acanthamoeba keratitis in Scotland: risk factors for contact lens wearers. Cont Lens Anterior Eye 22, 58-68.

Shoff, M., Rogerson, A., Schatz, S. \& Seal, D. (2007). Variable responses of Acanthamoeba strains to three multipurpose lens cleaning solutions. Optom Vis Sci 84, 202-207.

Shoff, M. E., Rogerson, A. \& Kessler, K. (2008). Prevalence of Acanthamoeba and other naked amoebae in South Florida domestic water. J Water Health 6, 99-104.

Stothard, D. R., Schroeder-Diedrich, J. M. \& Awwad, M. H. (1998). The evolutionary history of the genus Acanthamoeba and the identification of eight new $18 \mathrm{~S}$ rRNA gene sequence types. $J$ Eukaryot Microbiol 45, 45-54.

Vodkin, M. H., Howe, D. K. \& Visvesvara, G. S. (1992). Identification of Acanthamoeba at the generic and specific level using the polymerase chain reaction. J Protozool 39, 378-385.

Wynter-Allison, Z., Lorenzo-Morales, J. \& Calder, D. (2005). Acanthamoeba infection as a cause of severe keratitis in a soft contact lens wearer in Jamaica. Am J Trop Med Hyg 73, 92-94. 